\title{
In vitro performance of echoPIV for assessment of laminar flow profiles in a carotid artery stent
}

\author{
Astrid M. Hoving ${ }^{\text {a, } * \text { Jason Voorneveld, }}$ Julia Mikhal, ${ }^{\mathrm{c}}$ Johan G. Bosch, \\ Erik Groot Jebbink $\odot{ }^{d}$ and Cornelis H. Slump ${ }^{a}$ \\ ${ }^{a}$ University of Twente, TechMed Centre, Robotics and Mechatronics Group, Enschede, \\ The Netherlands \\ ${ }^{b}$ Erasmus MC, Thorax Center, Department of Biomedical Engineering, Rotterdam, \\ The Netherlands \\ ${ }^{c}$ University of Twente, TechMed Centre, BIOS Lab-on-a-Chip Group, Enschede, \\ The Netherlands \\ ${ }^{\mathrm{d} U n i v e r s i t y ~ o f ~ T w e n t e, ~ T e c h M e d ~ C e n t r e, ~ M u l t i-M o d a l i t y ~ M e d i c a l ~ I m a g i n g ~ G r o u p, ~}$ \\ Enschede, The Netherlands
}

\begin{abstract}
Purpose: Detailed blood flow studies may contribute to improvements in carotid artery stenting. High-frame-rate contrast-enhanced ultrasound followed by particle image velocimetry (PIV), also called echoPIV, is a technique to study blood flow patterns in detail. The performance of echoPIV in presence of a stent has not yet been studied extensively. We compared the performance of echoPIV in stented and nonstented regions in an in vitro flow setup.

Approach: A carotid artery stent was deployed in a vessel-mimicking phantom. High-frame-rate contrast-enhanced ultrasound images were acquired with various settings. Signal intensities of the contrast agent, velocity values, and flow profiles were calculated.

Results: The results showed decreased signal intensities and correlation coefficients inside the stent, however, PIV analysis in the stent still resulted in plausible flow vectors.

Conclusions: Velocity values and laminar flow profiles can be measured in vitro in stented arteries using echoPIV.

(C) The Authors. Published by SPIE under a Creative Commons Attribution 4.0 Unported License. Distribution or reproduction of this work in whole or in part requires full attribution of the original publication, including its DOI. [DOI: 10.1117/1.JMI.8.1.017001]
\end{abstract}

Keywords: high-frame-rate ultrasound; particle image velocimetry; carotid; stent; in vitro.

Paper 20116RR received May 6, 2020; accepted for publication Dec. 22, 2020; published online Jan. 13, 2021.

\section{Introduction}

Carotid artery stenosis and rupture of atherosclerotic plaque result in a high risk of stroke or transient ischemic attack. Therefore, in case a patient suffers from severe carotid artery stenosis, treatment is indicated. The plaque can be removed surgically, or a stent can be placed to reopen the artery during a minimally invasive procedure. Currently, surgery is preferred over stenting, due to the higher peri-procedural stroke risk of stenting compared with surgery. ${ }^{1,2}$ However, long-term outcomes of stenting are comparable with those of surgery. ${ }^{3,4}$ This suggests that technical developments to the peri-procedural process might improve the stenting procedure. Investigation into carotid artery stenting is therefore of interest.

Analysis and improvement of carotid artery stenting can be supplemented by investigation of possible blood flow pattern changes after stent insertion. Disturbed blood flow results in

*Address all correspondence to Astrid M. Hoving, a.m.hoving@utwente.nl 
decreased or oscillating wall shear stresses, leading to vessel wall remodeling. This increases the risk of atherosclerosis and stenosis. ${ }^{5}$ In case of stenting, the vessel wall remodeling caused by blood flow disturbances might result in in-stent stenosis. To capture the possible blood flow disturbances, detailed and quantitative information is required, provided by $2 \mathrm{D}$ or $3 \mathrm{D}$ vector fields obtained from data with high spatial and temporal resolution.

Investigation into imaging techniques to visualize detailed flow patterns in the presence of a stent is necessary, since current vascular imaging techniques have certain drawbacks. CT angiography does not provide velocity vector fields, and moreover it subjects the patient to harmful radiation and contrast agents. Magnetic resonance imaging suffers from strong metal artifacts in the area of the stent. ${ }^{6}$ Duplex ultrasound, the clinical standard to measure blood velocity by combining traditional and Doppler ultrasound, has an angle dependency, and flow information is limited to velocities in a specific sample volume inside the artery. ${ }^{7,8}$

To our knowledge, there are five ultrasound imaging techniques that are promising for studying flow patterns in a carotid artery stent. Vector Doppler, where two (or more) apertures are acquired to obtain both the axial and lateral velocity components, overcomes the limitation of Doppler ultrasound of measuring velocity in only one direction. ${ }^{9-12}$ Another technique applies transverse oscillations, i.e., perpendicular to the beam direction, to capture lateral components of the flow. ${ }^{13-15}$ An alternative, directional velocity estimation, is a technique where beam formation at multiple angles is used to find the angled frame with the highest correlation. ${ }^{16}$ Blood speckle tracking and echo-particle image velocimetry (echoPIV) are similar postprocessing strategies that can be applied to subsequent ultrasound frames to obtain velocity vectors. Both techniques divide the images into small blocks to estimate the mean displacement in each block based on chosen similarity index (usually cross-correlation or sum of squared differences). ${ }^{17,18}$ The main difference between blood speckle tracking and echoPIV is their signal source, where the former tracks the speckle patterns arising from red blood cell scattering, while echoPIV tracks intravenously injected microbubbles. ${ }^{19}$

From these five techniques, echoPIV seems ultimately suitable for imaging through a stent, since it uses ultrasound contrast agent (microbubbles) to enhance scattering of the moving fluid. The native blood scattering is very weak, resulting in low SNR. We expect that the stronger microbubble signal will overcome possible reduced signal intensity inside the stent.

EchoPIV has seen a considerable evolution since the first reported use of the technique in the medical field in $2004 .{ }^{19}$ In its early stage, several in vitro studies were performed to validate the technique, ${ }^{20-23}$ followed by in vivo studies in the left ventricle of the human heart. ${ }^{22-24}$ Due to the limited temporal resolution of the clinical ultrasound equipment, the frame rates were not sufficiently high to capture velocities greater than $0.4 \mathrm{~m} / \mathrm{s}$, which are present in ventricular flow. High frame rate (HFR) or ultrafast techniques overcome these problems. ${ }^{25}$ In 2015 , the use of plane wave imaging to achieve HFRs for echoPIV was described. ${ }^{26}$ Since then, flow patterns in the carotid artery and the left ventricle were studied in vitro ${ }^{27,28}$ and in vivo..$^{25,29-32}$ Moreover, HFR echoPIV was used in rabbits as proof of concept $^{26}$ and in humans to study abdominal aortic flow patterns. ${ }^{33,34}$

To our knowledge, the performance of echoPIV in the presence of a stent has not yet been reported other than in our previous preliminary study. ${ }^{35}$ In this paper, we compare the performance of HFR plane wave echoPIV in a stented region with a nonstented region in a flow phantom. In vitro flow studies are beneficial because of a controlled environment to change specific parameters to characterize the performance of the system. Signal intensities are expected to be lower in the stent than outside the stent, since the ultrasound wavefront will interfere with the metal struts of the stent. Since the thickness of the stent struts $(<100 \mu \mathrm{m})$ is smaller than the wavelength used this study (145 to $230 \mu \mathrm{m}$ ), no strong diffraction and interference patterns are expected inside the stent. Moreover, the openings of the stent mesh are larger $(>300 \mu \mathrm{m})$ than the ultrasound wavelength, so the ultrasound wavefront will not be blocked by the stent. The expectation is that the stent will have no significant impact on the resolved flow inside this type of stent. This might be different in stents with thicker struts or a denser mesh. However, the effects of different stent types is out of the scope of this study. The effect of one type carotid stent on in vitro echoPIV performance will be verified in this study. 


\section{Materials and Methods}

We evaluated the performance of echoPIV inside a stent in an in vitro flow setup. Several ultrasound acquisitions with variations from a single reference measurement were performed. Contrast ratios between stented and nonstented regions were obtained, and PIV analysis was performed.

\subsection{Experimental Setup}

Figure 1 shows a schematic representation of the experimental flow setup. A polyvinylalcoholgel (PVA-gel) phantom was fabricated using 10 weight\% PVA powder [Mw 85,000 to 124,000, $99+\%$ hydrolyzed (363146), Sigma-Aldrich, St. Louis, Missouri] and 90 weight $\%$ commercial cooling agent (Coolant $\mathrm{G} 12+/$ Longlife $-30^{\circ} \mathrm{C}$, AllRide). It was subjected to three freeze-thaw cycles of $17 \mathrm{~h}$ of freezing and $9 \mathrm{~h}$ of thawing. The phantom had a straight vessel design with a diameter of $8 \mathrm{~mm}$ and a length of $120 \mathrm{~mm}$. Due to shrinking after fabrication, the diameter was $7.2 \mathrm{~mm}$ when measurements were performed. A 100-cm straight tube with an inner diameter of $8 \mathrm{~mm}$ was placed in front of the phantom to ensure fully developed flow in the phantom. A commercial self-expanding stent (Wallstent $40 \times 10 \mathrm{~mm}$, Boston Scientific, Marlborough, Massachusetts) was halved to fit in the phantom and one half was deployed halfway the vessel. In the stented part, the diameter of the vessel was enlarged to $7.8 \mathrm{~mm}$ due to outward pointing radial force of the stent.

The flow setup was filled with a blood mimicking fluid (BMF) consisting of water and 39 mass $\%$ glycerin. Theoretically, this mixture has a kinematic viscosity of $3.3 \times 10^{-6} \mathrm{~m}^{2} / \mathrm{s}$ at $20^{\circ}$ C. The BMF was circulated through the setup using a gear pump with a continuous flow rate of $0.49 \mathrm{~L} / \mathrm{min}$ on average, based on values of carotid artery flow rate reported in the literature ${ }^{36-39}$ and adapted for the diameter of the phantom. The flow rate was measured by an ultrasonic flowmeter (UF Ultrasonics Flow Meter, Cynergy3 Components, United Kingdom, 3\% accuracy). The contrast agent, BR-14 microbubbles powder (Bracco Suisse SA, Geneva, Switzerland) was mixed by hand with $0.4-\mathrm{mL}$ demineralized water, was added to $2 \mathrm{~L} \mathrm{BMF}$.

\subsection{Ultrasound Measurement Protocol}

Ultrasound acquisition was performed using a Verasonics Vantage 256 system (Verasonics Inc., Kirkland, Washington) with a Verasonics L12-3v linear array transducer. Two overlapping subapertures of 128 elements of the total of 192 elements were used for each angled plane wave acquisition (this was required because the probe was fitted with a single 128 channel interface to the Vantage system). In total, 250 frames were acquired per dataset. Using the reference settings (first row of Table 1), an uncompounded frame rate of 8789 frames per second was achieved (pulse repetition interval of individual frames was $113.78 \mu \mathrm{s}$ ). Automatic singular value decomposition (SVD) filtering was applied to suppress semistationary signals, while retaining the signal from the moving microbubbles. ${ }^{33}$ The angled frames were coherently compounded after SVD-filtering. No contrast-specific sequences such as pulse inversion or amplitude modulation were used.

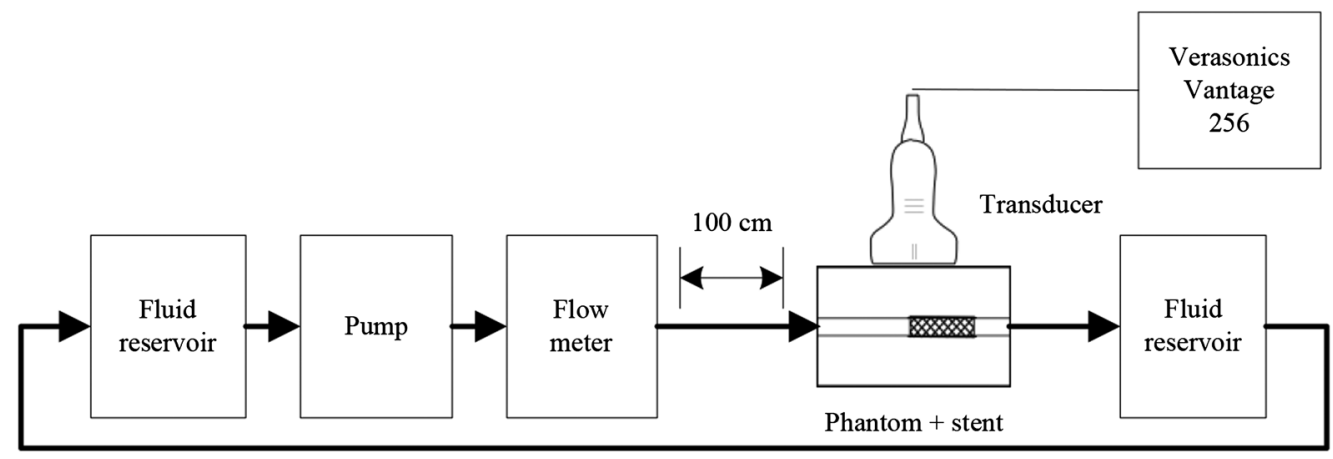

Fig. 1 Schematic representation of the experimental flow setup. 
Hoving et al.: In vitro performance of echoPIV for assessment of laminar flow profiles...

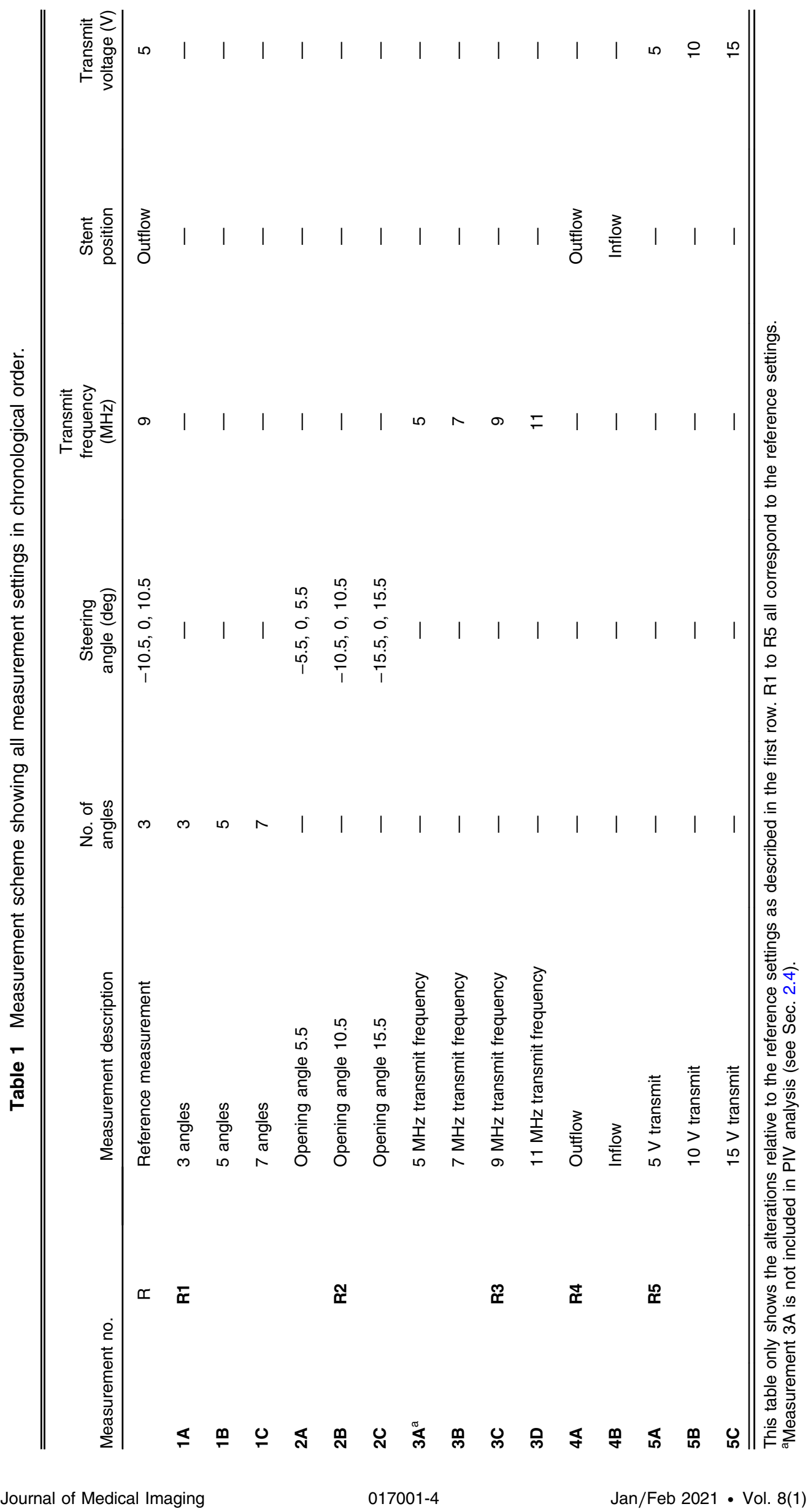


Table 1 shows the settings of all acquisitions. We performed 15 measurements in 30 min, while BMF was continuously flowing without adding new contrast-agent. Within these 15 measurements, five subcategories can be distinguished. In each subcategory, one of the settings was changed, i.e., in the first category only the number of steering angles was altered. Each subcategory contained one measurement with settings equal to the so-called reference setting (Table 1, first row). We altered the number of steering angles, the angulation of the plane waves, the transmit frequency, and the transmit voltage. The ultrasound probe was positioned such that vessel was in the field of view with the stent located at the outflow side of the vessel (referred to as outflow in Table 1, column 6), except for measurement 4B, where we changed the position of the stent to the inflow side of the vessel (referred to as inflow in Table 1, column 6).

\subsection{Analysis: Contrast Between Nonstented and Stented Regions}

The contrast ratio between the signal power inside and outside the stent was calculated. We manually selected a region of interest (ROI) both inside and outside the stent. Figure 2 shows that the ROIs are located at the same depth and with an equal distance from the center in the lateral direction. Although this figure shows the ROIs on the B-mode images, contrast is calculated using the IQ-data of the reconstructed, concatenated SVD-filtered frames. Contrast is calculated as

$$
\text { Contrast }(\mathrm{dB})=10 \log _{10}\left(\frac{P_{\text {nostent }}}{P_{\text {stent }}}\right) \text {, }
$$

where the power outside the stented area is $P_{\text {nostent }}=I_{\text {nostent }}^{2}+Q_{\text {nostent }}^{2}$ and the power inside the stent is $P_{\text {stent }}=I_{\text {stent }}^{2}+Q_{\text {stent }}^{2} I$ and $Q$ result from the IQ-data.

Contrast values were averaged over 250 frames.

\subsection{Analysis: Particle Image Velocimetry}

PIV analysis was performed in a modified version of PIVlab ${ }^{40}$ in MATLAB R2017b (The Mathworks Inc., Natick, Massachusetts) on the IQ-data of the compounded SVD-filtered frames. Two iterative interrogation areas of $32 \times 32$ pixels and $16 \times 16$ pixels with an overlap of $75 \%$

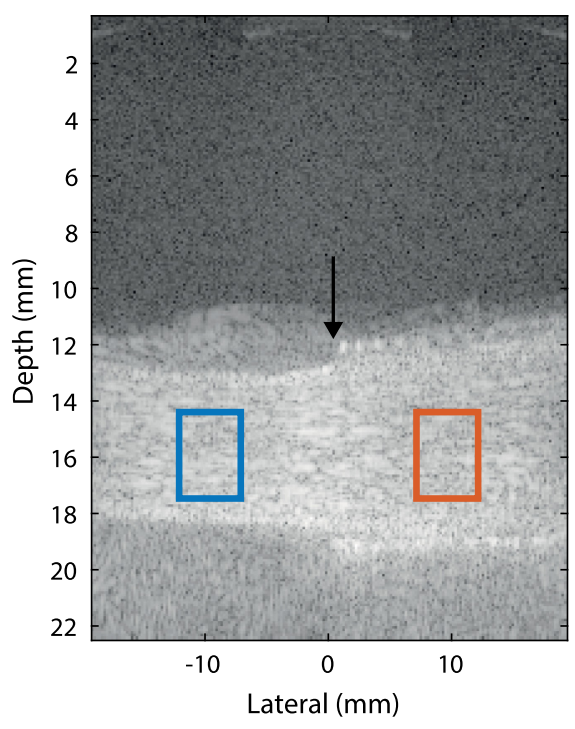

Fig. 2 Time-averaged SVD-filtered B-mode frame of reference measurement 1 (R1) showing the ROls of the nonstented region (blue) and stented region (orange) for contrast analysis. The stented area is from lateral position $0 \mathrm{~mm}$ to the right border of the image. The black arrow indicates the start of the stent. 
were applied on the first 100 frames of each measurement. Due to these interrogation areas, it is required to have pixels surrounding the ROI. In measurement $3 \mathrm{~A}$, the imaging depth was not sufficient to have the required number of pixels surrounding the contrast-enhanced vessel, therefore, PIV analysis could not be completed on measurement 3A (5 MHz transmit frequency). Outliers were removed and further postprocessing consisted of smoothing using Gaussian filters and moving averages.

Mean and peak velocity and correlation coefficients were computed and analyzed. The velocity data and correlation coefficients of each measurement were split into two separate regions: nonstented and stented (see Fig. 5). The vectors and correlation coefficients inbetween the two regions were not included, to prevent ambiguity about which region the vectors belonged to.

\subsection{Statistical Analysis: Contrast}

Within each subcategory ( 1 to 5 ), mean contrast values were compared. Also, mean contrast values of the reference measurements were compared with each other.

A one-way ANOVA was conducted in SPSS (IBM Corp. Released 2019. IBM SPSS Statistics for Windows, Version 26.0. Armonk, New York: IBM Corp.) to compare contrast values between measurements with 3, 5, or 7 angles, with different steering angles, with 5, 7, 9, and $11 \mathrm{MHz}$ transmit frequencies, with 5,10, and $15 \mathrm{~V}$ transmit voltage and between the reference measurements. Tukey post hoc tests were conducted on these measurements to evaluate the individual differences. An independent samples T-test was conducted in SPSS to compare the measurements with the stent position on the inflow and on the outflow side.

\subsection{Statistical Analysis: PIV}

Statistical analysis on PIV results was performed on the five measurements that have equal settings, the reference measurements. PIV analysis resulted in estimated velocity vectors over 98 timeframes. For mean velocity calculation, all velocity magnitudes per region (stented and nonstented) were averaged over each frame. For maximum velocity, the maximum velocity magnitudes of each lateral position per region were averaged over each frame. This resulted in 98 mean and 98 maximum velocity magnitudes per region for each measurement.

Mean and maximum velocities outside the stent were compared with velocities in the stented region using independent-samples T-tests in SPSS. Mean and maximum velocities of each frame were taken as input. Correlation coefficients in and outside the stent were compared using the same approach.

Mean velocity inside the stent was compared with the theoretical mean velocity using onesample T-test in SPSS. The axial theoretical velocity profile, derived from the Hagen-Poiseuille equation, can be described as

$$
v(r)=v_{c}\left(1-\frac{r^{2}}{R^{2}}\right)
$$

where $v(r)$ is the velocity at radius $r$ of the total vessel radius $R$, and $v_{c}$ is the center velocity. The equation for the theoretical mean velocity in the ultrasound image plane was derived by integrating Eq. (2) and dividing it by the diameter of the vessel:

$$
v_{\text {mean }}=\frac{v_{c}}{2 R} \int_{-R}^{+R}\left(1-\frac{r^{2}}{R^{2}}\right) \mathrm{d} r=\frac{2}{3} v_{c} .
$$

We assumed $v_{c}$ to be the maximum velocity, which is calculated as

$$
2 \times\left(\frac{Q}{\pi R^{2}}\right),
$$

where $Q$ is the flow rate measured by the flow meter. 
Using Eqs. (2)-(4), the theoretical mean and maximum velocity in the nonstented region (diameter is $7.2 \mathrm{~mm}$ ) are 26.7 and $40.1 \mathrm{~cm} / \mathrm{s}$, respectively. The theoretical mean and maximum velocity in the stented region (diameter is $7.8 \mathrm{~mm}$ ) are 22.8 and $34.2 \mathrm{~cm} / \mathrm{s}$, respectively.

\section{Results}

\subsection{Contrast Between Nonstented and Stented Regions}

Mean contrast had a positive value in all measurements, so the intensity was higher in the nonstented region than in the stented part of the vessel (Fig. 3). Since mean contrast values were small (in the range of 0 to $3 \mathrm{~dB}$ ), this difference was hardly visible in the time-averaged SVD frames (Fig. 2). The measurements using transmit voltage of 5 and $15 \mathrm{~V}$ showed a large spread compared with all other measurements.

Figure 3 shows boxplots of the contrast measurements where statistically significant differences within subgroups are indicated. One-way ANOVA showed a statistically significant difference $(p<0.001)$ in contrast value within each subgroup. Increasing the number of angles resulted in statistically significant lower contrast, thus less difference between nonstented and stented areas. A steering angle of $15.5 \mathrm{deg}$ shows lowest contrast value, compared with 5.5- and 10.5-deg steering angles. Transmit frequency had less influence on intensity between the two areas. The contrast was significantly lower when the stent was positioned on the outflow side compared with the inflow side. In the transmit voltage subgroup, the lowest contrast was seen using 5 or $10 \mathrm{~V}$ compared with $15 \mathrm{~V}$.

The reference measurements were also significantly different $(p=0.012)$. Tukey's post hoc test revealed that only reference measurements one and five differed significant $(p=0.006)$ (Fig. 4).

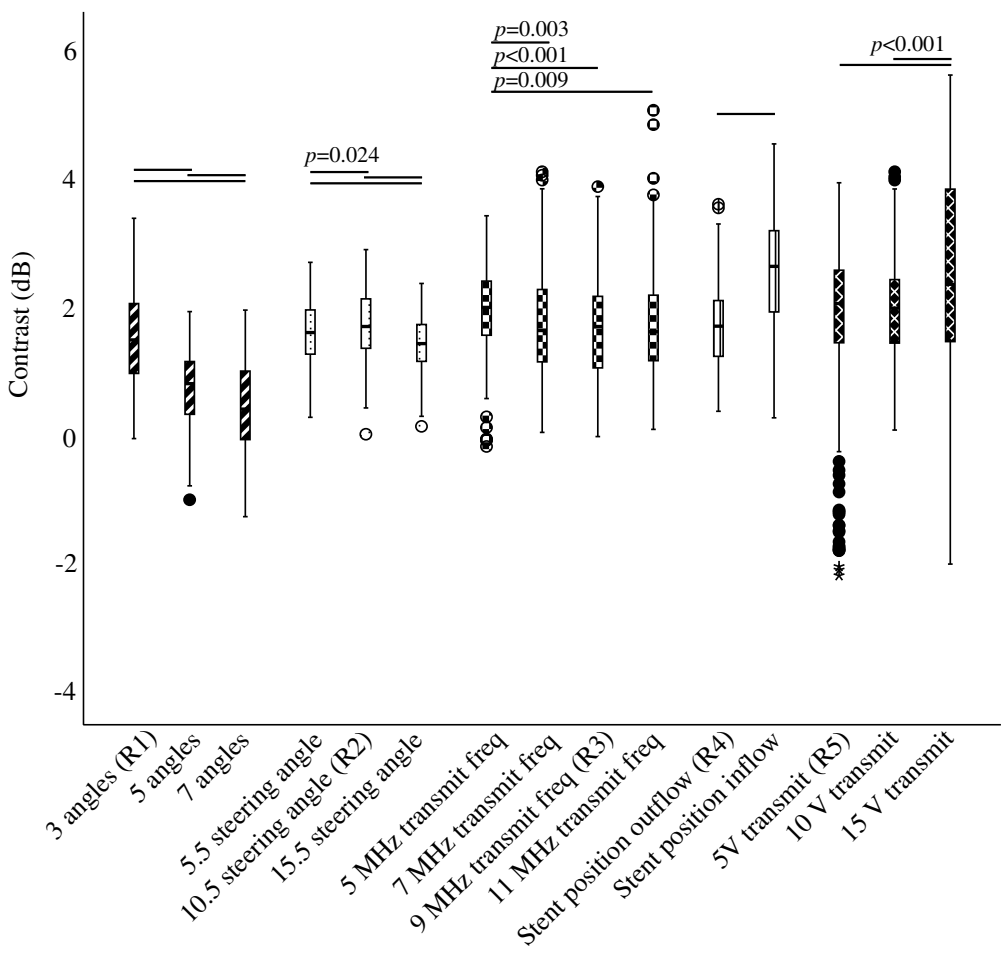

Fig. 3 Boxplots of the contrast in all measurements. The different subcategories are clustered. Statistical differences within subcategories are indicated with lines above specific measurements. $p \ll 0.001$ applies for all comparisons, unless $p$-values are noted above the lines. 


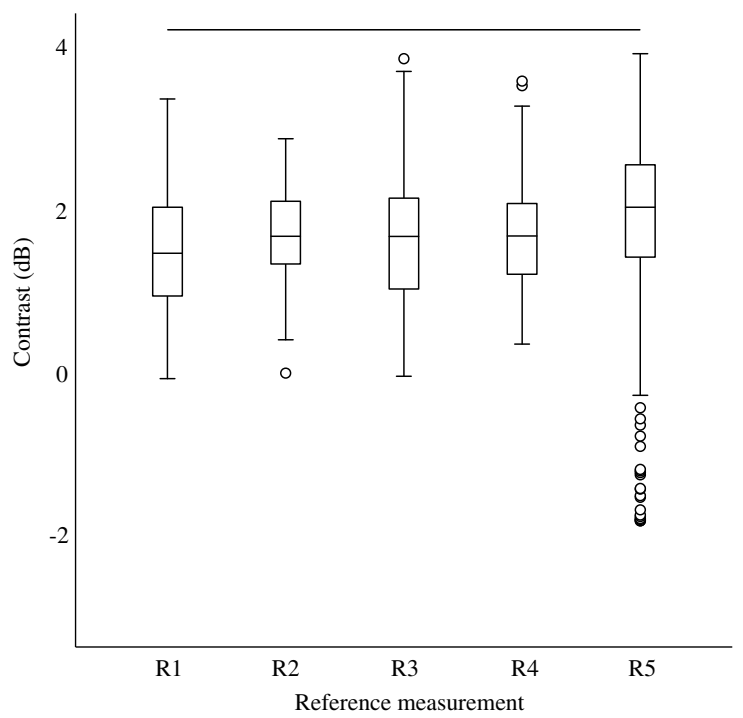

Fig. 4 Boxplots of the contrast in the reference measurements. The line above R1 to R5 shows the statistically significant difference between these two measurements $(p=0.006)$.

\subsection{Particle Image Velocimetry}

PIV analysis showed laminar flow patterns in the entire vessel (Fig. 5). Notice the increased diameter in the stented region. Mean and maximum velocities were lower in the stented region than in the nonstented region (Table 2). The difference between the two regions was larger for the mean velocity $(4.32 \mathrm{~cm} / \mathrm{s})$ than for the maximum velocity $(2.58 \mathrm{~cm} / \mathrm{s})$. Correlation coefficients were consistently lower in stented region (0.72) than in nonstented region (0.82) (Table 2).

PIV-derived velocities were compared with theoretical values. The mean velocity inside the stent $(22.48 \mathrm{~cm} / \mathrm{s}, \mathrm{SD}=0.435)$ was significantly different from the theoretical mean velocity $(22.8 \mathrm{~cm} / \mathrm{s}) ; p \ll 0.001$.

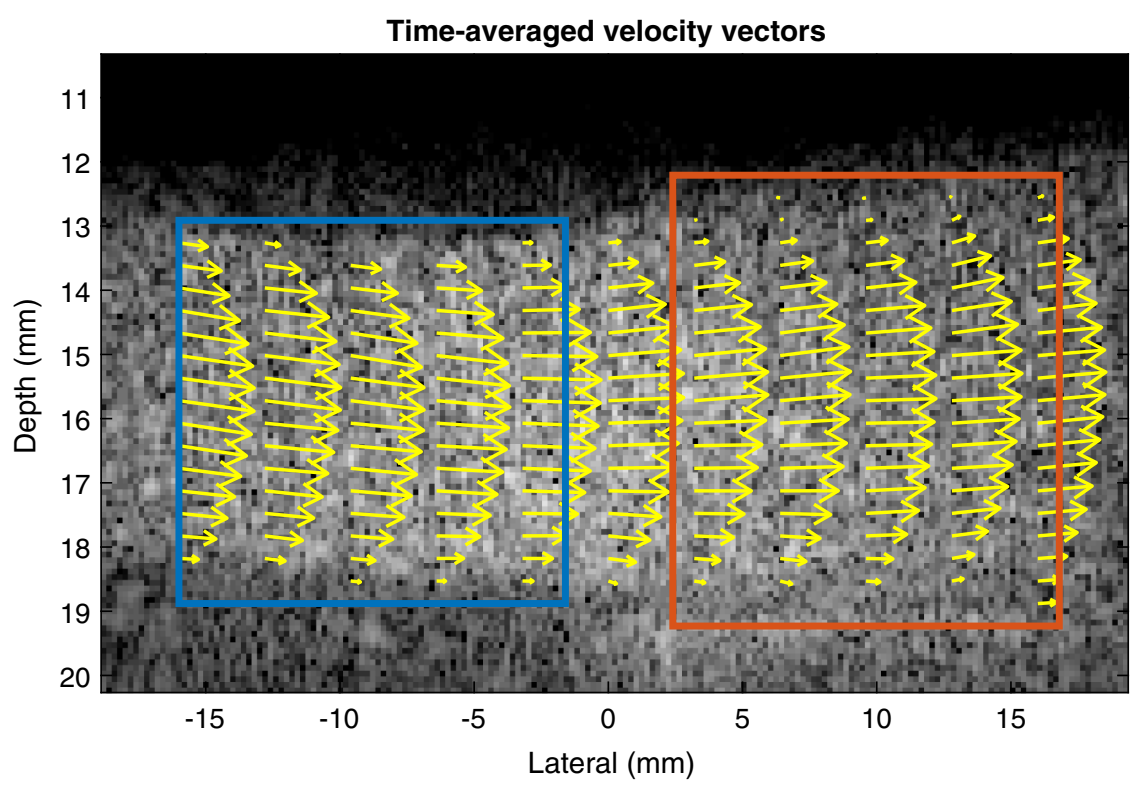

Fig. 5 Time-averaged velocity vectors (yellow arrows) of reference measurement 1 in nonstented area (blue) and stented area (orange). The velocity vectors are projected on a single SVD filtered frame. 
Hoving et al.: In vitro performance of echoPIV for assessment of laminar flow profiles...

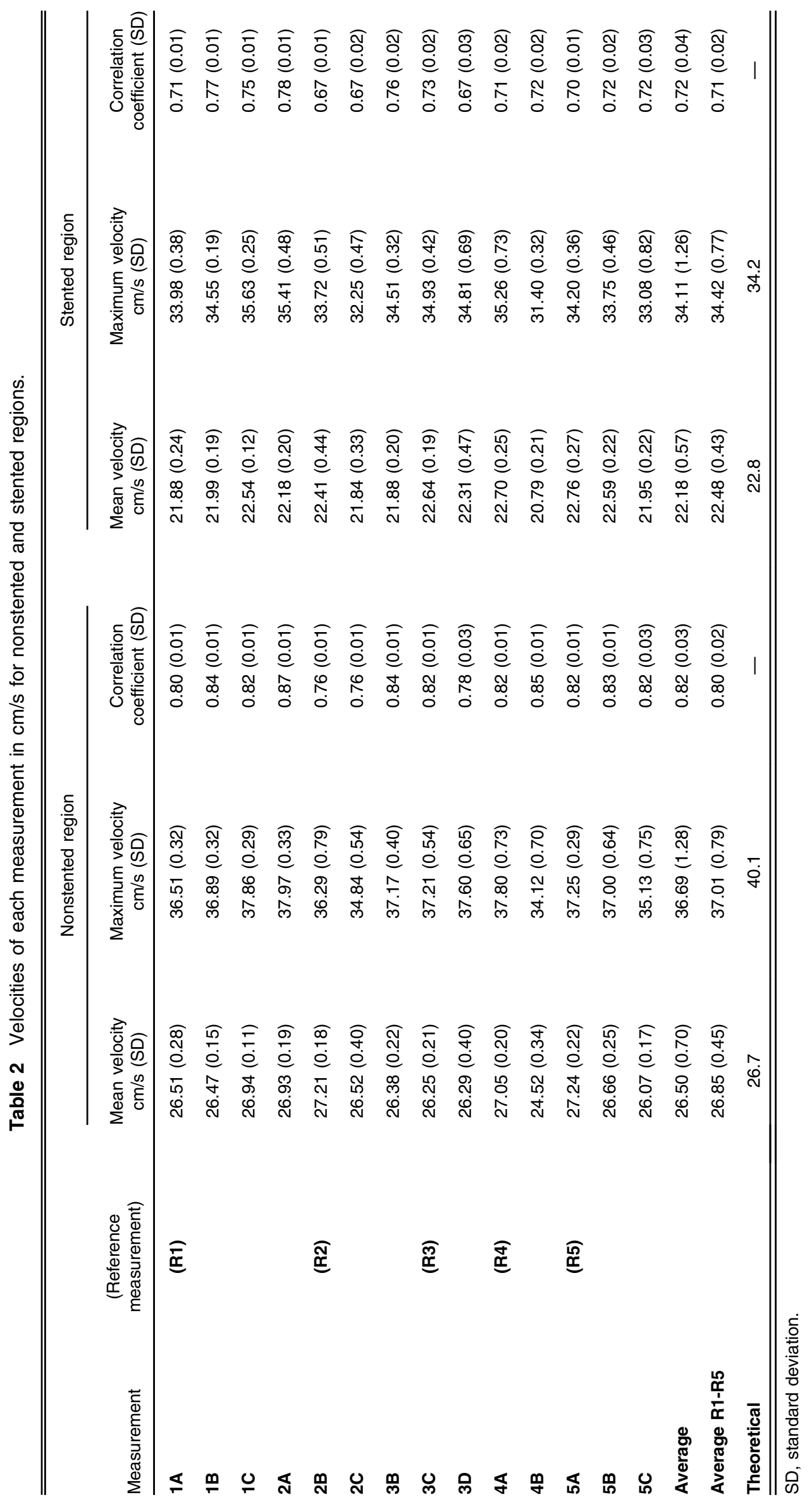


(a)

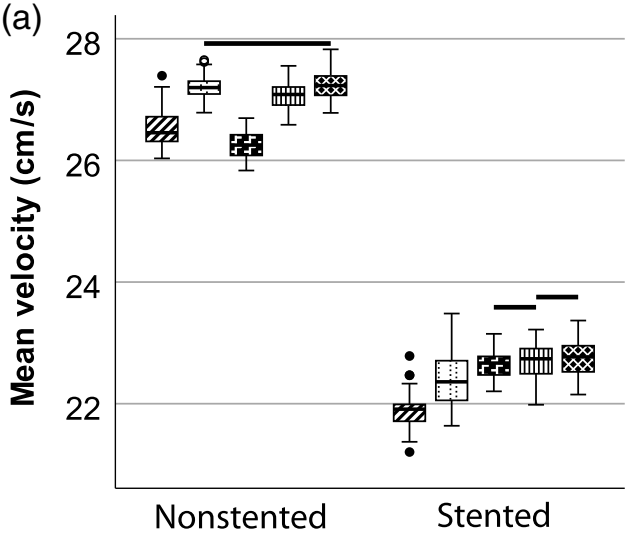

(c)

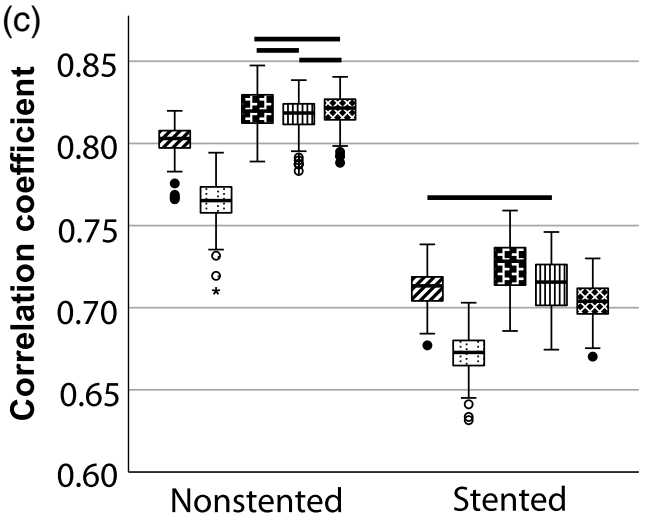

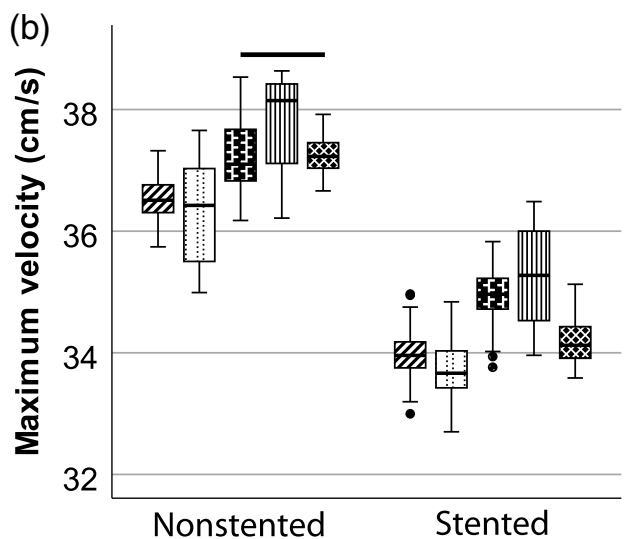

Measurement

UR 1

R2

5: $\mathrm{R} 3$

IIIIIR4

R5

Fig. 6 Boxplots of reference measurements showing (a) mean velocities, (b) maximum velocities, and (c) correlation coefficients for nonstented and stented regions. The horizontal lines above the boxplots indicate no significant difference $(p>0.05)$.

Comparison of the five reference measurements resulted in significant differences in mean and maximum velocities and in correlation coefficients. Some measurements were not statistically significantly different as depicted the boxplots of Fig. 6.

\section{Discussion}

In this study, we have evaluated the effect of a carotid artery stent on contrast agent signal intensity and the accuracy of echoPIV-derived velocities inside the stented region. To evaluate the difference in signal intensity between the stented and nonstented regions, the contrast between these two regions was calculated. The contrast analysis shows that, on average, signal intensity is lower in the stented region than outside the stent. PIV analysis resulted in lower velocity values and correlation coefficients in the stented region than in the nonstented region. To analyze the difference from a ground truth, the velocity in the stent was compared with an estimated theoretical value. A statistically significant difference was seen between mean velocity in the stent and the theoretical mean velocity.

This study is a continuation of our previous preliminary experiments on the effect of a stent on HFR echoPIV. ${ }^{35}$ To the best of our knowledge, these are the first studies that report about HFR echoPIV in a stent. EchoPIV is an emerging technique to study blood flow patterns in detail, and it might be ultimately suitable for analysis of in vivo stent performance, because the enhanced scattering of the moving fluid might overcome a possible reduction of image quality caused by the stent. The setup has been improved since the previous study. We now used a linear array transducer with a shorter elevation depth and a better ultrasound-eligible phantom material, to overcome the limitations reported in that study. For this study, we have added the analysis of 
several settings on the effect of imaging through a stent and elaborated on contrast and velocity estimations. The decreased correlation coefficients and signal intensities in the stent are consistent with data obtained in our previous study. ${ }^{35}$

We conclude that contrast measurements are reproducible over time, however, the results of velocity analysis seem not that reproducible. This is based on the five measurements with equal settings, the so-called reference measurements. Contrast was only significantly different between reference measurements 1 and 5 (Fig. 4), while the results of PIV analysis showed significant differences between most reference measurements (Fig. 6). A possible explanation for this lack of consistency is the variability in flow control, as the pump operated within a range of 0.46 and $0.52 \mathrm{~L} / \mathrm{min}$. Moreover, contrast is a ratio of two intensity values, while velocity and correlation coefficient are absolute values, which are more prone to subtle changes.

From literature and previous experiments, we know that microbubbles are disrupted by the pressure of the ultrasound beam..$^{33,41,42}$ Theoretically, the microbubble signal would be lower at the outflow side of the vessel, because the microbubbles are exposed longer to the ultrasound beam. In our reference setup, the stent is positioned at the outflow side of the vessel, therefore we cannot distinguish if a lower signal strength in the stented part would be caused by the stent or by disrupted bubbles. Measurement 4 is performed to check this. If bubbles were measurably destroyed during one measurement, we would expect to see a lower value for contrast in measurement $4 \mathrm{~B}$ (stent on the inflow side). In that case, the signal intensity in the stent would be higher (more bubbles), and the signal intensity outside the stent would be lower (less bubbles). However, our results show a significantly increased contrast value in measurement 4B. Therefore, we do not assume that the difference in contrast between the two regions in all other measurements are due to destroyed bubbles at the outflow side. The decreased correlation coefficients in the stented part, also present in measurement 4B, confirm these thoughts.

There are differences between stented and nonstented regions in both contrast measurements and PIV outcomes. On average, the contrast has a positive value, which means that the signal intensity is lower in the stent than outside the stent. This is a logical result of imaging through a stent, because imaging through a grid of metal wire can attenuate the ultrasound waves, resulting in less enhanced signal in the stented region. Mean and maximum velocities are also lower in the stented region of the phantom. Since the diameter of the phantom was increased by the forces of the stent, we expected to see lower velocities in the stent. Due to this diameter difference, it is difficult to determine if the metal wires of the stent also contributed to the decrease in velocity estimation. Correlation coefficients were also significantly lower in the stented region. As correlation coefficients indicate quality of PIV analysis, we can conclude that quality of velocity estimation decreases when imaging through a stent. However, the quality of PIV in a stented vessel is acceptable, since PIV analysis still showed laminar flow profiles and plausible velocity values.

PIV settings, such as the size of the interrogation area, should be carefully chosen. These settings depend on, for example, expected velocities, pixel size, and point spread function. We used two iterative interrogation windows of $32 \times 32$ pixels and $16 \times 16$ pixels, commonly used sizes and 2D Gaussian peak fitting as subpixel displacement estimation method. The maximum velocity of $\sim 40 \mathrm{~cm} / \mathrm{s}$ and the effective frame rate of $\sim 3 \mathrm{kHz}$ results in a displacement of $\sim 0.7$ pixel per frame. This satisfies the design rule of a displacement less than a quarter of the interrogation area. ${ }^{43}$ However, the downside of subpixel displacement is that the results are more prone to error. Possible solutions to improving accuracy would be skipping frames for PIV analysis (increasing the effective frame-to-frame displacement to over 1 pixel) or increasing the resolution of the beamforming grid (decreasing pixel size-however, this would incur higher computation costs).

Comparison with theoretical velocities and profiles is difficult in this set of measurements. Mean velocity in the stented region differed significantly from the theoretical value. It turns out that comparison with a theoretical profile is complicated, because the field of view is probably not in the center of the vessel. This is based on the observation that the diameter in the images does not correspond to the real diameter. Moreover, the slice thickness, and thereby averaging of velocities, contributes to the differences with theoretical velocities. Due to these differences, we cannot define a certain offset for velocities obtained by echoPIV imaging through a stent. However, there is also no definition of such a deviation or correction factor for clinical 
Duplex in carotid stents. Some studies showed increased peak systolic velocities in the stent, ${ }^{44-46}$ whereas others showed no difference ${ }^{47}$ or stent-dependent deviations. ${ }^{48}$

A large variance in contrast is visible in measurements $5 \mathrm{~A}$ and $5 \mathrm{C}$ of the transmit voltage subcategory, with standard deviations of 1.25 and $1.59 \mathrm{~dB}$, respectively (Fig. 3). In comparison, the pooled standard deviation of all contrast measurements is $0.97 \mathrm{~dB}$. The larger spread in these two measurements could be due to alterations to the setup because of changing the position of the stent in measurement 4B. However, measurement 5B has a standard deviation of $0.74 \mathrm{~dB}$, which is significantly lower and does not support this explanation for large spread. Another explanation might be bubble disruption, as we know this occurs at higher transmit voltages. ${ }^{33,41,42}$ The increased contrast in the measurement of $15 \mathrm{~V}$ transmit voltage can be explained by the severe bubble disruption, as we expect to see lower signal intensity at the outflow side of the vessel after bubble disruption at high pressure. Also, during the measurement, bubble concentration will become lower, this can explain the larger spread in the measurement. We see an increase of contrast value over time for measurement $5 \mathrm{C}$; however, this trend is not present in other measurements (Appendix, Fig. 7). Therefore, we cannot draw conclusions on the role of bubble disruption in the larger spread at higher transmit voltages.

Our measurements show a decrease in contrast values when the number of angles increases (measurements 1A-C, Fig. 3). If the stent is seen as an obstruction to the ultrasound signal, then approaching the vessel from different angles would result in less signal loss. The more angles are used, the more signal will reach the bubbles, and therefore lower contrast values will be seen.

A limitation of this study is the restriction to one stent type. Future in vitro studies should focus on the effects of HFR echoPIV in other stent types, since the cell areas (open structures of the stents) differ between different stent types. This may influence the ultrasound signal protrusion through the stent. Another limitation is the restricted comparison with the theoretical flow profiles. As indicated before, the possible cause of the deviation from the theoretical flow profile, especially in the nonstented part, is the positioning of the ultrasound transducer. This will remain a critical and challenging issue in future experiments. However, since the diameter of the vessel was enlarged by the stent and the transition zone was short, we do not know for sure if there was a fully developed flow profile in the stent. The stent itself, creating a "rough surface," will also influence the flow profile. To investigate the effect of the metal of the stent on the ultrasound performance, the stent should ideally not change the diameter nor the surface roughness of the vessel. For comparison with in vivo situations, pulsatile flow conditions and comparison with theoretical Womersley profiles are highly recommended. Besides the pulsatile flow, in vivo situations are far more complex than this simplified ideal and straight vessel without bifurcation, which may also be of influence of the performance of echoPIV in in vivo stents.

\section{Conclusions}

This study shows that velocity profiles in the presence of a stent can be measured using echoPIV in an in vitro setup. The stent causes a decrease in signal intensities of contrast agent. This results in decreased correlation coefficients after PIV analysis. Both observations correspond to our expectations that a Wallstent causes the image quality to decrease. However, our results show that the quality with a correlation coefficient of 0.7 is still sufficient to perform PIV analysis. Velocity vectors of continuous flow within clinical range of carotid flow rates $(0.49 \mathrm{~L} / \mathrm{min})$ can be obtained in a stented phantom. Future work should focus on pulsatile flow conditions and in vivo effects of stent on the quality of echoPIV.

\section{Appendix A: Contrast Values Over Time}

We visualized contrast for each frame separately, to check whether contrast increases because of bubble disruption. Bubble disruption occurs at higher transmit voltages. Therefore, measurement 5 is of interest to see this effect. Figure 7 shows an increase in contrast over time in measurement $5 \mathrm{C}$ (transmit voltage $15 \mathrm{~V}$ ). However, a decrease over time is visible in measurement $5 \mathrm{~B}$ (transmit voltage $10 \mathrm{~V})$. 


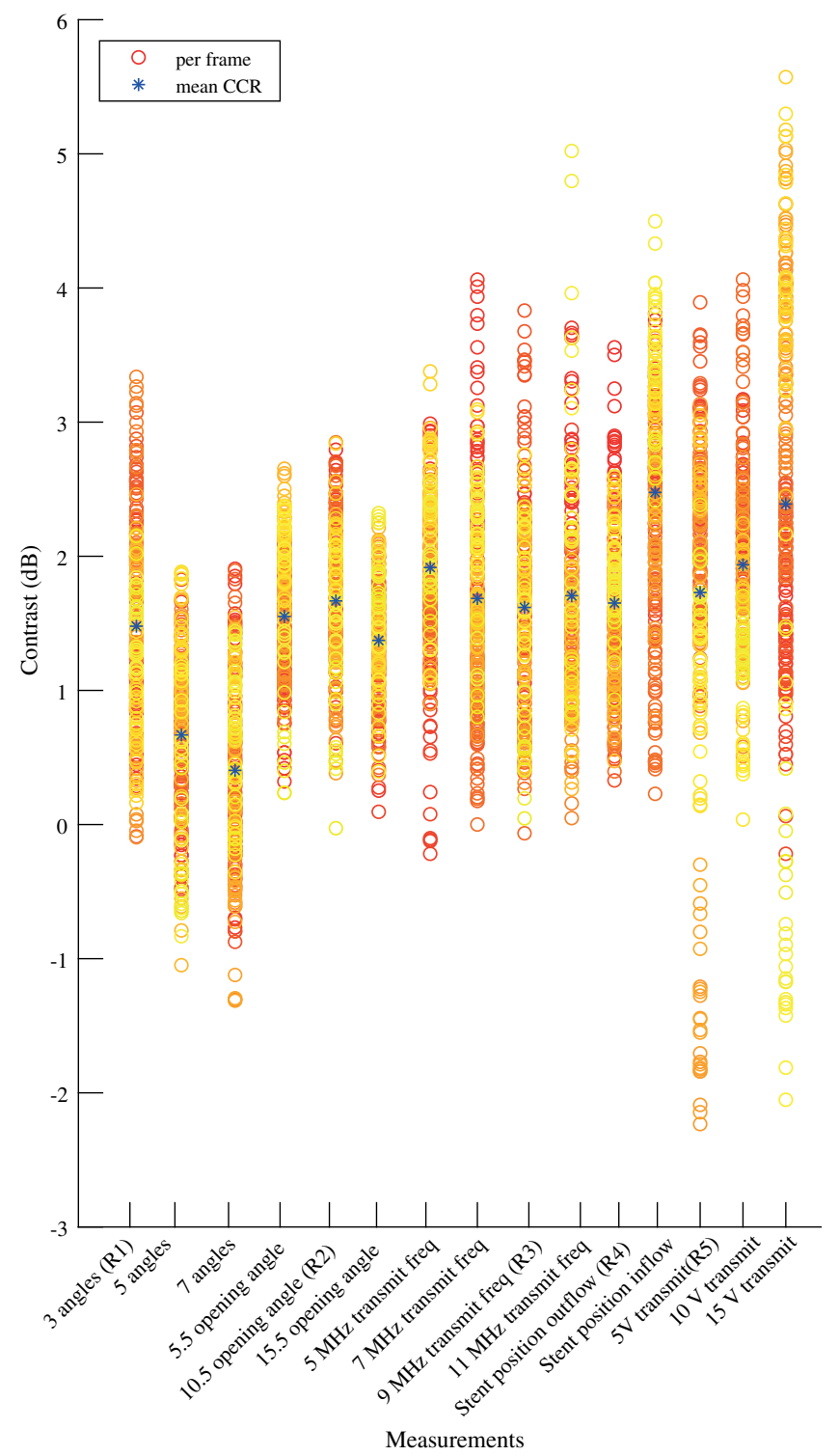

Fig. 7 Scatterplot of contrast values in $\mathrm{dB}$ per measurement. Each circle represents a contrast measurement of one frame. The color scale ranges from red [timepoint $1(=0 \mathrm{~s})$ ] to yellow [latest timepoint $(=0.17 \mathrm{~s})]$.

\section{Disclosures}

Previous work on this subject is published in a SPIE medical imaging conference proceedings. ${ }^{35}$ No conflicts of interest, financial or otherwise, are declared by the authors.

\section{Acknowledgments}

This research was financially supported by the Stichting TWIN (Foundation TWIN). The authors state no conflict of interest and have nothing to disclose. The authors would like to thank Bracco Suisse SA for providing BR14 microbubbles. Thanks to Henny Kuipers for his contributions to the design and fabrication of the flow setup, BMPI group for the use of their ultrasound equipment, and thanks to the ultrasound experts of Erasmus MC Thoraxcenter for the detailed technical help and feedback. 


\section{References}

1. T. G. Brott et al., "Stenting versus endarterectomy for treatment of carotid-artery stenosis," N. Engl. J. Med. 363(1), 11-23 (2010).

2. H. Bonati et al., "Percutaneous transluminal balloon angioplasty and stenting for carotid artery stenosis," Cochrane database Syst. Rev. 9(9), CD000515 (2012).

3. G. J. De Borst and A. R. Naylor, "In the end, it all comes down to the beginning!" Eur. J. Vasc. Endovasc. Surg. 50(3), 271-272 (2015).

4. L. H. Bonati et al., "Long-term outcomes after stenting versus endarterectomy for treatment of symptomatic carotid stenosis: the International Carotid Stenting Study (ICSS) randomised trial," Lancet 385(9967), 529-538 (2015).

5. J. J. Wentzel et al., "Geometry guided data averaging enables the interpretation of shear stress related plaque development in human coronary arteries," J. Biomech. 38(7), 1551-1555 (2005).

6. T. Klemm et al., "MR imaging in the presence of vascular stents: a systematic assessment of artifacts for various stent orientations, sequence types, and field strengths," J. Magn. Reson. Imaging 12(4), 606-615 (2000).

7. M. Y. Park et al., "Effect of beam-flow angle on velocity measurements in modern Doppler ultrasound systems," Am. J. Roentgenol. 198(5), 1139-1143 (2012).

8. J. D. Acker et al., "Duplex carotid ultrasound," Neuroradiology 28, 608-617 (1986).

9. M. D. Fox, "Multiple crossed-beam ultrasound Doppler," IEEE Trans. Sonics Ultrason. 25(5), 281-286 (1978).

10. B. Dunmire et al., "Cross-beam vector Doppler ultrasound for angle-independent velocity measurements," Ultrasound Med. Biol. 26(8). 1213-1235 (2000).

11. S. Ricci, L. Bassi, and P. Tortoli, "Real-time vector velocity assessment through multigate Doppler and plane waves," IEEE Trans. Ultrason. Ferroelectr. Freq. Control 61(2), 314-324 (2014).

12. S. Ricci et al., "Real-time blood velocity vector measurement over a 2-D region," IEEE Trans. Ultrason. Ferroelectr. Freq. Control 65(2), 201-209 (2018).

13. J. A. Jensen and P. Munk, "A new method for estimation of velocity vectors," IEEE Trans. Ultrason. Ferroelectr. Freq. Control 45(3), 837-851 (1998).

14. S. Salles et al., "2-D arterial wall motion imaging using ultrafast ultrasound and transverse oscillations," IEEE Trans. Ultrason. Ferroelectr. Freq. Control 62(6), 1047-1058 (2015).

15. M. Lenge et al., "Plane-wave transverse oscillation for high-frame-rate 2-D vector flow imaging," IEEE Trans. Ultrason. Ferroelectr. Freq. Control 62(12), 2126-2137 (2015).

16. J. Kortbek and J. A. Jensen, "Determination of velocity vector angles using the directional cross-correlation method," IEEE Trans. Ultrason. Ferroelectr. Freq. Control 53(11), 2036-2049 (2006).

17. G. E. Trahey, J. W. Allison, and O. T. Von Ramm, "Angle independent ultrasonic detection of blood flow," IEEE Trans. Biomed. Eng. BME-34(12), 965-967 (1987).

18. L. N. Bohs et al., "Speckle tracking for multi-dimensional flow estimation," Ultrasonics 38(1), 369-375 (2000).

19. H. B. Kim, J. R. Hertzberg, and R. Shandas, "Development and validation of echo PIV," Exp. Fluids 36(3), 455-462 (2004).

20. L. Liu et al., "Development of a custom-designed echo particle image velocimetry system for multi-component hemodynamic measurements: system characterization and initial experimental results," Phys. Med. Biol. 53(5), 1397-1412 (2008).

21. J. Westerdale et al., "Flow velocity vector fields by ultrasound particle imaging velocimetry," J. Ultrasound Med. 30(2), 187-195 (2011).

22. C. Prinz et al., "Can echocardiographic particle image velocimetry correctly detect motion patterns as they occur in blood inside heart chambers? A validation study using moving phantoms," Cardiovasc. Ultrasound 10(1), 1-10 (2012).

23. A. Kheradvar et al., "Echocardiographic particle image velocimetry: a novel technique for quantification of left ventricular blood vorticity pattern," J. Am. Soc. Echocardiogr. 23(1), 86-94 (2010). 
24. H. Abe et al., "Contrast echocardiography for assessing left ventricular vortex strength in heart failure: a prospective cohort study," Eur. Heart J. Cardiovasc. Imaging 14(11), 1049-1060 (2013).

25. J. Voorneveld et al., "High-frame-rate echo-particle image velocimetry can measure the high-velocity diastolic flow patterns," Circ. Cardiovasc. Imaging 12(4), e008856 (2019).

26. C. H. Leow et al., "Flow velocity mapping using contrast enhanced high-frame-rate plane wave ultrasound and image tracking: methods and initial in vitro and in vivo evaluation," Ultrasound Med. Biol. 41(11), 2913-2925 (2015).

27. C. H. Leow and M. X. Tang, "Spatio-temporal flow and wall shear stress mapping based on incoherent ensemble-correlation of ultrafast contrast enhanced ultrasound images," Ultrasound Med. Biol. 44(1), 134-152 (2018).

28. J. Voorneveld et al., "High frame rate ultrasound particle image velocimetry for estimating high velocity flow patterns in the left ventricle," IEEE Trans. Ultrason. Ferroelectr. Freq. Control 65(12), 2222-2232 (2017).

29. L. Nie et al., "High-frame-rate contrast-enhanced echocardiography using diverging waves: 2-D motion estimation and compensation," IEEE Trans. Ultrason. Ferroelectr. Freq. Control 66(2), 359-371 (2019).

30. M. E. G. Toulemonde et al., "High frame-rate contrast echocardiography: in-human demonstration," JACC Cardiovasc. Imaging 11(6), 923-924 (2018).

31. P. E. Gates et al., "Measurement of wall shear stress exerted by blowing blood in the human carotid artery: ultrasound Doppler velocimetry and echo particle image velocimetry," Ultrasound Med. Biol. 44(7), 1392-1401 (2018).

32. A. Gurung et al., "Echo particle image velocimetry for estimation of carotid artery wall shear stress: repeatability, reproducibility and comparison with phase-contrast magnetic resonance imaging," Ultrasound Med. Biol. 43(8), 1618-1627 (2017).

33. J. Voorneveld et al., "High-frame-rate contrast-enhanced ultrasound for velocimetry in the human abdominal aorta," IEEE Trans. Ultrason. Ferroelectr. Freq. Control 65(12), 2245-2254 (2018).

34. S. Engelhard et al., "High-frame-rate contrast-enhanced US particle image velocimetry in the abdominal aorta: first human results," Radiology 289(1), 119-125 (2018).

35. A. M. Hoving et al., "In vitro high-frame-rate contrast-enhanced ultrasound particle image velocimetry in a carotid artery stent," Proc. SPIE 10580, 105800A (2018).

36. M. Cibis et al., "Wall shear stress calculations based on 3D cine phase contrast MRI and computational fluid dynamics: a comparison study in healthy carotid arteries," NMR Biomed. 27(7), 826-834 (2014).

37. M. Schoning, J. Walter, and P. Scheel, "Estimation of cerebral blood flow through color duplex sonography of the carotid and vertebral arteries in healthy adults," Stroke 25(1), 17-22 (1994).

38. S. W. Lee et al., "Geometry of the carotid bifurcation predicts its exposure to disturbed flow," Stroke 39(8), 2341-2347 (2008).

39. Y. Hoi et al., "Characterization of volumetric flow rate waveforms at the carotid bifurcations of older adults," Physiol. Meas. 31(3), 291-302 (2010).

40. W. Thielicke and E. J. Stamhuis, "PIVlab - towards user-friendly, affordable and accurate digital particle image velocimetry in MATLAB," J. Open Res. Software 2, e30 (2014).

41. M. Toulemonde, R. J. Eckersley, and M. X. Tang, "High frame rate contrast enhanced echocardiography: microbubbles stability and contrast evaluation," in IEEE Int. Ultrason. Symp. (2017).

42. O. Couture, M. Fink, and M. Tanter, "Ultrasound contrast plane wave imaging," IEEE Trans. Ultrason. Ferroelectr. Freq. Control 59(12), 2676-2683 (2012).

43. R. D. Keane and R. J. Adrian, "Optimization of particle image velocimeters. Part I: Double pulsed systems," Meas. Sci. Technol. 1, 1202-1215 (1990).

44. B. K. Lal et al., "Duplex ultrasound velocity criteria for the stented carotid artery," J. Vasc. Surg. 47(1), 63-73 (2008).

45. S. F. Stanziale et al., "Determining in-stent stenosis of carotid arteries by duplex ultrasound criteria," J. Endovasc. Ther. 12(3), 346-353 (2005). 
46. A. R. Naylor et al., "Management of atherosclerotic carotid and vertebral artery disease: 2017 clinical practice guidelines of the ESVS," Eur. J. Vasc. Endovasc. Surg. 55(1), 3-81 (2018).

47. F. T. M. Bosch et al., "Optimal cut-off criteria for duplex ultrasound compared with computed tomography angiography for the diagnosis of restenosis in stented carotid arteries in the international carotid stenting study," Eur. Stroke J. 2(1), 37-45 (2017).

48. C. Spies et al., "Carotid artery stent type influences duplex ultrasonography derived peak systolic velocity: findings of an in-vitro model," Catheter. Cardiovasc. Interv. 70(2), 309-315 (2007).

Astrid M. Hoving received her BSc and MSc degrees in technical medicine from the University of Twente in Enschede, The Netherlands, in 2012 and 2016, respectively. She started in 2016 as a PhD student at the University of Twente at the Robotics and Mechatronics group in collaboration with Vascular Department of the University Medical Centre in Utrecht, The Netherlands. Her doctoral research focuses on imaging of the carotid arteries, more specifically in vitro flow studies using ultrasound and stenosis degree measurements on CTA.

Jason Voorneveld received his BSc degree in electromechanical engineering and MSc degree in biomedical engineering from the University of Cape Town, South Africa. He then completed his $\mathrm{PhD}$ at the Erasmus Medical Center in Rotterdam, The Netherlands, where he now works as a postdoc. His research interests include high frame rate ultrasound imaging, ultrasound contrast agents, 3D ultrasound imaging, and blood flow estimation.

Julia Mikhal received her PhD from the University of Twente in 2012. She is a postdoctoral fellow at the University of Twente, pursuing the study of precision stenting of intracranial and carotid arteries in the context of a personal VENI grant (2015) of the Dutch Foundation for Scientific Research. She is an expert in numerical methods and large-scale simulation. She works on vascular disease models combining mathematical modeling and analysis with highperformance computing.

Johan G. Bosch received his MSc degree electrical engineering from Eindhoven University of Technology, Netherlands, in 1985, and PhD from Leiden University Medical Center, Netherlands, in 2006. He is an associate professor at the Department of Biomedical Engineering, Thoraxcenter, Erasmus MC, Rotterdam, Netherlands. He is a (co-)PI of projects on 3-D ultrasound image formation, transducer development, 2D and 3D cardiovascular imaging, flow and tissue stiffness assessment using novel ultrasound approaches. His research interests include echocardiographic image processing, transducer development, and ultrasound techniques for image formation and functional imaging.

Erik Groot Jebbink received his BS and MS degrees in technical medicine from the University of Twente, Enschede, The Netherlands, in 2009 and 2013, respectively. In 2017, he received his $\mathrm{PhD}$ from the Physics of Fluids group, University of Twente. He is currently an assistant professor at the M3i group, University of Twente and Technical Physician at the Department of Vascular Surgery at the Rijnstate Hospital, Arnhem. His research interests focus on the interaction between blood flow and stents, based on in vitro and in vivo measurements. He received a Dutch NWO-ZonMW-VENI personal grant in 2020.

Cornelis H. Slump is a professor at the University of Twente. He graduated TU Delft in electrical engineering in 1979 and obtained his $\mathrm{PhD}$ in theoretical physics from Rijksuniversiteit Groningen in 1984. In 1983, he joined Philips Medical Systems, group leader x-ray predevelopment cardiovascular. He was as an assistant professor in the University of Twente, Department of Electrical Engineering, Digital Signal Processing group in 1989. He served as Dean of Education from 1994 to 1998 and full professor in 1999. His research interests are functional medical imaging and image analysis. 